\title{
Chiral and herringbone symmetry breaking in water-surface monolayers
}

\author{
I. R. Peterson* \\ Institut für Physikalische Chemie, Johannes Gutenberg-Universität, Jakob Welder-Weg 11, D-55099 Mainz, Germany \\ R. M. Kenn, A. Goudot, P. Fontaine, and F. Rondelez \\ Institut Curie PC-PSI, 11, rue Pierre et Marie Curie, F-75231 Paris Cedex 05, France \\ W. G. Bouwman and K. Kjaer \\ Physics Department, Risø National Laboratory, DK-4000 Roskilde, Denmark \\ (Received 6 September 1994; revised manuscript received 13 January 1995)
}

\begin{abstract}
We report the observation from monolayers of eicosanoic acid in the $L_{2}^{\prime}$ phase of three distinct outof-plane first-order diffraction peaks, indicating molecular tilt in a nonsymmetry direction and hence the absence of mirror symmetry. At lower pressures the molecules tilt in the direction of their nearest neighbors. In this region we find a structural transition, which we tentatively identify as the rotatorherringbone transition $L_{2 d}-L_{2 h}$.

PACS number(s): 61.30.Gd, 68.15. $+\mathrm{e}, 64.70 . \mathrm{Ja}, 68.18 .+\mathrm{p}$
\end{abstract}

\section{INTRODUCTION}

Monolayers of amphiphiles are important in colloids, detergents, and biological membranes [1]; as an essential step in the fabrication of Langmuir-Blodgett films [2]; and as a system of choice for investigating the structure and thermodynamics of systems of low dimensionality $[3,4]$. Since the exact computation by Onsager of the thermodynamic properties of the Ising model, it has been known that phase transitions, impossible in onedimensional systems, are possible in two dimensions. Nelson and Halperin [3,4] have shown more recently that, in addition to analogs of bulk gaseous, liquid, and crystalline solid phases, two-dimensional phases may display a previously unrecognized type of organization. This organization, called hexatic, is therefore characteristic for lamellar systems. A rich polymorphism in monolayer systems was in fact reported long ago by StällbergStenhagen and Stenhagen [5]; however experimental and conceptual difficulties have meant that its validity has only recently been confirmed by using grazing-incidence $x$-ray diffraction (GID) [6,7]. Hexatic monolayer phases have now been unequivocally demonstrated [8,9], and they include the room-temperature monolayer phases of many biologically and technologically interesting amphiphiles.

One related topic that has attracted attention for a long time is the influence of chirality on monolayer thermodynamics [10-12]. Chiral monolayer packings have been reported in two recent atomic-force-microscope studies of monolayers deposited on solid substrates $[13,14]$. In the first of these, the substance was the racemate of an unusual ladder molecule, and the observations

\footnotetext{
${ }^{*}$ Present address: Nima Technology, The Science Park, Coventry CV4 7EZ, United Kingdom.
}

were interpreted as its demixing into pure enantiomers. The second report [14], involving a nonchiral molecule, associated the chirality with the presence of specific metallic counterions. Here we report GID measurements indicating the existence in water-surface monolayers of a chiral packing of nonchiral molecules in the absence of counterions. The measurements also indicate the existence of a phase transition, which we tentatively identify as the herringbone-rotator transition.

\section{EXPERIMENT}

The present measurements were made over a 24-h period at Beamline BW1 at HASYLAB, DESY, Hamburg, Germany. The measurement setup has been described previously [15-17]. Eicosanoic acid ( $>99 \%$ pure) was purchased from Aldrich and made up into approximately 1-mM spreading solutions using P.A. grade chloroform from Merck. The subphase was in all cases ultrapure water, purified by using a Millipore desktop unit.

A monolayer was spread at the beginning of the measurement period and all the measurements were carried out on this monolayer without respreading. A hydrophilic slab under the monolayer damped capillary waves induced by mechanical vibrations. The horizontally propagating fan beam from the synchrotron was confined vertically, using slits so as to illuminate slightly less than the full trough width, and deflected downward using a quartz mirror at 0.85 of the angle for total external reflection. At regular intervals the vertical position of the trough was adjusted to center the beam laterally, and if the specular reflectivity fell below 0.96 , the water level relative to the hydrophilic slab was adjusted.

The trough was moved horizontally every few hours to expose new areas of the monolayer to the beam. The horizontal distribution of the diffracted $x$ rays was determined by scanning a Soller collimator (consisting of many 
parallel vertical plates), and their vertical distribution was determined using a vertically resolving linear position-sensitive detector mounted behind the Soller collimator in the plane of incidence and normal to the line joining the center of the beam footprint on the water surface and the detector center [18]. The accumulated position-resolved counts were corrected for polarization, footprint, powder-averaging (Lorentz factor), and distance effects. The Yoneda-Vineyard peak [19] was used to determine the detector position corresponding to the waterline, but otherwise all values within twice the angle of total external reflection were discarded.

The whole set of corrected intensities was leastsquares-fitted to a sum of model peaks paired symmetrically above and below the plane of the water surface, plus a linear background. Each model peak was Lorentzian parallel to the water and Gaussian normal to it [20]. In the case of three peaks, the $z$ coordinates of the peaks were restricted by the relationship $Q_{z 1}=Q_{z 2}+Q_{z 3}$, where peak 1 is the one with the largest $Q_{z}$. In the case of only two peaks, the restriction was changed to either $Q_{z 1}=2 Q_{z 2}$ or $Q_{z 2}=0$, as appropriate. These two possibilities correspond to molecular tilt in next-nearest-neighbor (NNN) or nearest-neighbor (NN) directions, respectively [21].

\section{RESULTS}

The nine different surface conditions investigated, together with their best-fit peak parameters, are listed in Table I. Three qualitatively different diffraction patterns were observed. Typical examples of each are shown in Figs. 1-3, which are plotted on the same scale to simplify comparison. Each is a contour plot of the corrected intensities as a function of the scattering vector components $Q_{x y}$ (abscissa) and $Q_{z}$ (ordinate). The set of all best-fit parameters from all the surface conditions is given in the table.

The plot of Fig. 1 corresponds to the run at $3.5 \mathrm{mN} / \mathrm{m}$ and $21.1^{\circ} \mathrm{C}$. Two peaks can be seen, one of them with its maximum essentially in the plane of the water surface, characteristic for tilt in the direction of the nearest neighbor, just as reported for docosanoic acid under similar conditions. The peak intensity of the in-plane peak is approximately twice that of the out-of-plane peak.

On the assumption of two Gaussian-Lorentzian peaks, one in the plane of the water surface, the normalized value of $\chi^{2}$ for the best fit to this data set was 2.20. A fit with three distinct peaks originating from the one crystal packing led to no significant decrease of the best-fit $\chi^{2}$. Similar results were obtained from two other measurement runs at low surface pressures but different temperatures. Nevertheless, there was a significant qualitative difference found in the low-pressure data sets. Figure 2 corresponds to $4.8 \mathrm{mN} / \mathrm{m}$ and $24.4{ }^{\circ} \mathrm{C}$. It can be seen that the in-plane peak in this case is much less intense.

Figure 3 shows a contour plot of the corrected intensities from the run at $14.9 \mathrm{mN} / \mathrm{m}$ and $25.7^{\circ} \mathrm{C}$. Three peaks, labeled $A, B$, and $C$, are clearly evident. Three tests were performed to check whether peak $A$ at (1.43, 0.50 ) could have originated from a collapsed monolayer. First, the best-fit normalized $\chi^{2}$ in both cases was calcu-

TABLE I. Best-fit peak parameters for all surface conditions investigated: temperature T; surface pressure $\pi$; peak intensity $I$; peak coordinates $Q_{x y}$ and $Q_{z}$ and their widths.

\begin{tabular}{|c|c|c|c|c|c|c|c|}
\hline $\begin{array}{c}\mathrm{T} \\
\left({ }^{\circ} \mathrm{C}\right) \\
\end{array}$ & $\begin{array}{c}\pi \\
(\mathrm{mN} / \mathrm{m}) \\
\end{array}$ & $\begin{array}{c}I \\
\text { (arb. units) }\end{array}$ & $\begin{array}{c}Q_{x y} \\
\left(\operatorname{rad~\AA ^{-1}}\right) \\
\end{array}$ & $\begin{array}{c}Q_{z} \\
\left(\operatorname{rad~} \AA^{-1}\right) \\
\end{array}$ & $\begin{array}{c}\Delta Q_{x y} \\
(\mathrm{FWHM}) \\
\left(\operatorname{rad} \AA^{-1}\right)\end{array}$ & $\begin{array}{c}\Delta Q_{z} \\
\left(\mathrm{FWHM}^{-1}\right) \\
\left(\operatorname{rad} \AA^{-1}\right)\end{array}$ & $\chi^{2}$ \\
\hline \multirow[t]{2}{*}{15.8} & 4.5 & 491 & 1.459 & 0.000 & 0.018 & 0.27 & 2.44 \\
\hline & & 304 & 1.436 & 0.642 & 0.049 & 0.26 & \\
\hline \multirow[t]{2}{*}{21.1} & 3.5 & 708 & 1.462 & 0.000 & 0.016 & 0.28 & 2.20 \\
\hline & & 364 & 1.391 & 0.713 & 0.046 & 0.26 & \\
\hline \multirow[t]{2}{*}{24.4} & 4.8 & 66 & 1.474 & 0.000 & 0.012 & 0.25 & 1.30 \\
\hline & & 339 & 1.427 & 0.630 & 0.040 & 0.26 & \\
\hline \multirow[t]{2}{*}{31.4} & 5.4 & 39 & 1.477 & 0.000 & 0.019 & 0.41 & 1.30 \\
\hline & & 327 & 1.421 & 0.587 & 0.036 & 0.26 & \\
\hline \multirow[t]{3}{*}{23.8} & 14.9 & 463 & 1.487 & 0.036 & 0.027 & 0.26 & 1.58 \\
\hline & & 446 & 1.479 & 0.451 & 0.037 & 0.25 & \\
\hline & & 39 & 1.449 & 0.415 & 0.137 & 0.61 & \\
\hline \multirow[t]{3}{*}{25.7} & 14.9 & 513 & 1.492 & 0.071 & 0.027 & 0.29 & 2.05 \\
\hline & & 516 & 1.479 & 0.428 & 0.032 & 0.26 & \\
\hline & & 99 & 1.427 & 0.499 & 0.041 & 0.27 & \\
\hline \multirow[t]{3}{*}{27.4} & 14.9 & 477 & 1.492 & 0.025 & 0.023 & 0.29 & 1.79 \\
\hline & & 526 & 1.474 & 0.442 & 0.030 & 0.26 & \\
\hline & & 55 & 1.431 & 0.478 & 0.051 & 0.34 & \\
\hline \multirow[t]{3}{*}{29.4} & 14.9 & 404 & 1.493 & 0.087 & 0.022 & 0.31 & 1.59 \\
\hline & & 490 & 1.473 & 0.424 & 0.030 & 0.27 & \\
\hline & & 156 & 1.426 & 0.511 & 0.016 & 0.30 & \\
\hline \multirow[t]{3}{*}{31.3} & 15.0 & 348 & 1.493 & 0.111 & 0.022 & 0.20 & 1.76 \\
\hline & & 412 & 1.469 & 0.419 & 0.034 & 0.29 & \\
\hline & & 296 & 1.426 & 0.531 & 0.010 & 0.15 & \\
\hline
\end{tabular}




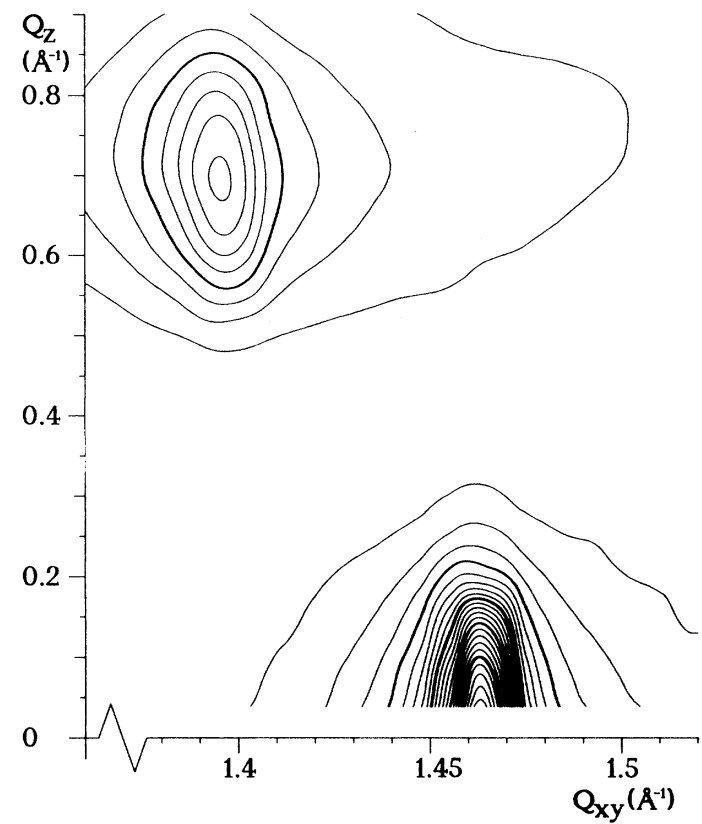

FIG. 1. Contour plot of the corrected diffraction intensity vs in-plane and out-of-plane scattering vector components $Q_{x y}$ and $Q_{z}$ radians. $\AA^{-1}$ at $3.5 \mathrm{mN} / \mathrm{m}$ and $21.1^{\circ} \mathrm{C}$.

lated. On the assumption that peak $A$ is a collapsedmonolayer peak, $\chi^{2}$ was 3.04 , whereas on the assumption that the three peaks originate from the one packing, the value decreased to 2.12 . Second, the peak widths were considered. The best-fit widths (full width at half maximum, FWHM), of the $A$ peak were $(0.04,0.26)$, which is quite comparable to those of the other two peaks, whereas a collapsed monolayer should give significantly

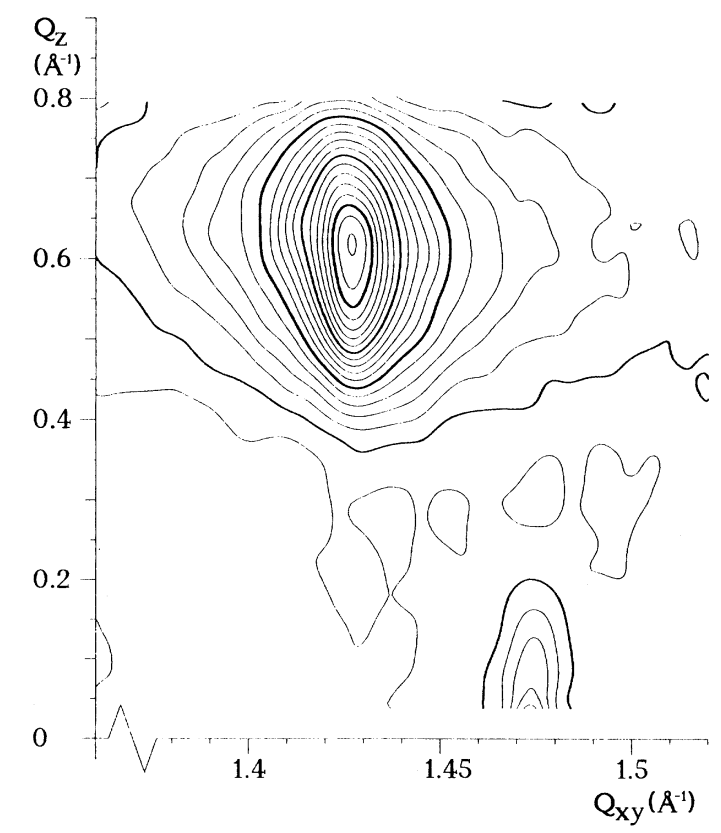

FIG. 2. Contour plot of the corrected diffraction intensity vs in-plane and out-of-plane scattering vector components $Q_{x y}$ and $Q_{z}$ radians. $\AA^{-1}$ at $4.8 \mathrm{mN} / \mathrm{m}$ and $24.4^{\circ} \mathrm{C}$.

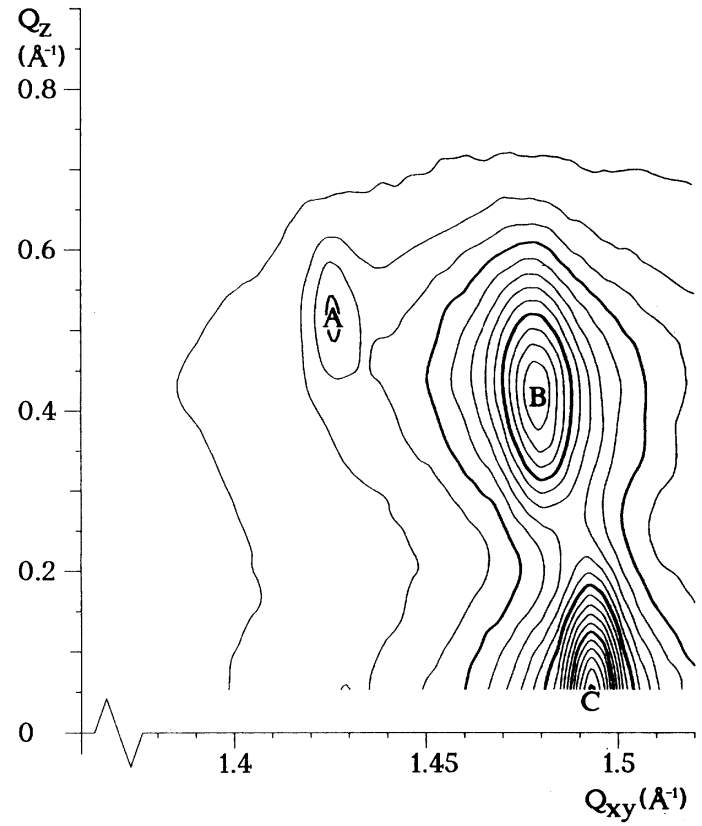

FIG. 3. Contour plot of the corrected diffraction intensity vs in-plane and out-of-plane scattering vector components $Q_{x y}$ and $Q_{z}$ radians. $\AA^{-1}$ at $14.9 \mathrm{mN} / \mathrm{m}$ and $25.7^{\circ} \mathrm{C}$.
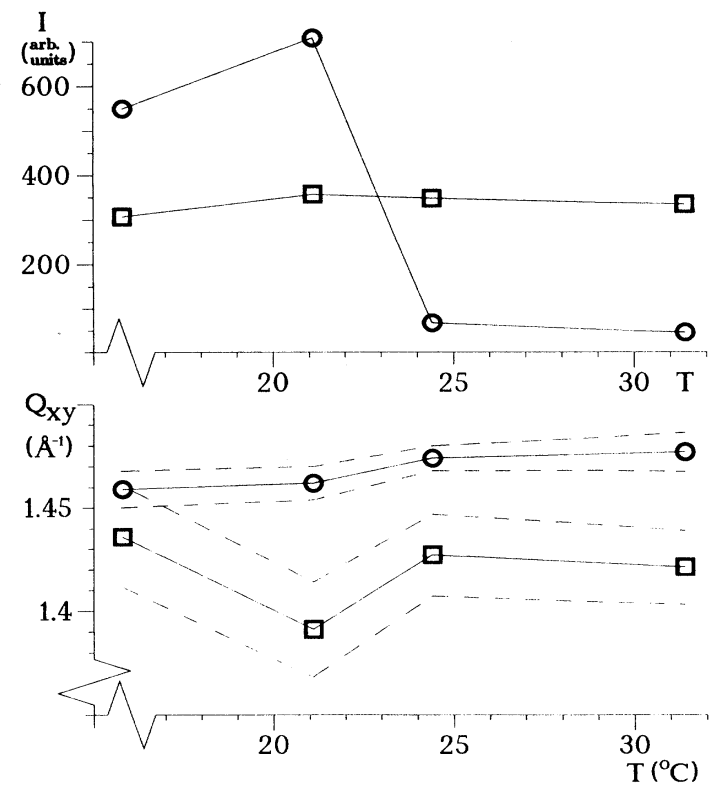

FIG. 4. Plots of the peak intensity $I$ and in-plane component $Q_{x y}$ of the scattering vector as a function of temperature $\mathrm{T}$ for each of the two peaks in the low-surface-pressure series: circles, in-plane (nondegenerate) peak; squares, out-of-plane (degenerate) peak. The dashed lines in the $Q_{x y}$ plot indicate the halfmaximum points. The worst-case standard deviation of the nonsystematic error due to Poisson counting statistics in the upper $I$ plot is equal to the symbol line thickness; and in the lower $Q_{x y}$ plot, equal to one-quarter of the graph line thickness. 
TABLE II. Lattice and tilt parameters extracted from Table I for all surface conditions investigated.

\begin{tabular}{ccccccr}
\hline $\begin{array}{c}\mathrm{T} \\
\left({ }^{\circ} \mathrm{C}\right)\end{array}$ & $\begin{array}{c}\pi \\
(\mathrm{mN} / \mathrm{m})\end{array}$ & $\begin{array}{c}A_{\text {xs }} \\
\left(\AA^{2}\right)\end{array}$ & $\theta$ & \multicolumn{2}{c}{ Tilt } & \multicolumn{2}{c}{ Distortion } \\
15.8 & 4.5 & 19.377 & 27.649 & 0 & 0.09962 & 0 \\
21.1 & 3.5 & 19.479 & 31.099 & 0 & 0.09035 & 0 \\
24.4 & 4.8 & 19.575 & 26.784 & 0 & 0.07061 & 0 \\
31.4 & 5.4 & 19.661 & 19.433 & 0 & 0.05949 & 0 \\
23.8 & 14.9 & 19.912 & 21.841 & 3 & 0.0455 & 7 \\
25.7 & 14.9 & 19.859 & 17.329 & 8 & 0.03508 & 17 \\
27.4 & 14.9 & 19.861 & 18.317 & 6 & 0.03546 & 17 \\
29.4 & 14.9 & 19.851 & 17.068 & 11 & 0.02916 & 13 \\
31.3 & 15.0 & 19.833 & 16.663 & 15 & 0.02568 & 5 \\
\hline \hline
\end{tabular}

smaller peak widths: the greater thickness should lead directly to narrower width in the $z$ direction and indirectly, via the higher dimensionality, to longer-range order in the plane. For example, in the case of docosanoic acid [22] the widths are $(0.007,0.1)$. Finally, since the equilibrium spreading pressure of fatty acids on water corresponds essentially to the expanded-to-condensed transition, which in docosanoic acid near ambient temperature occurs below $1 \mathrm{mN} / \mathrm{m}$, a collapsed monolayer is expected to remain collapsed upon reducing the pressure. However, the third peak disappeared without a trace upon reducing the surface pressure to $5 \mathrm{mN} / \mathrm{m}$.

Figure 4 shows the variation of peak intensities and in-plane peak positions for the four low-surface-pressure data sets showing NN tilt. Between the conditions of Figs. 1 and 2, the intensity of the in-plane peak changes by a factor of more than 10 over a $3-\mathrm{K}$ temperature range. The discontinuity in this parameter is most
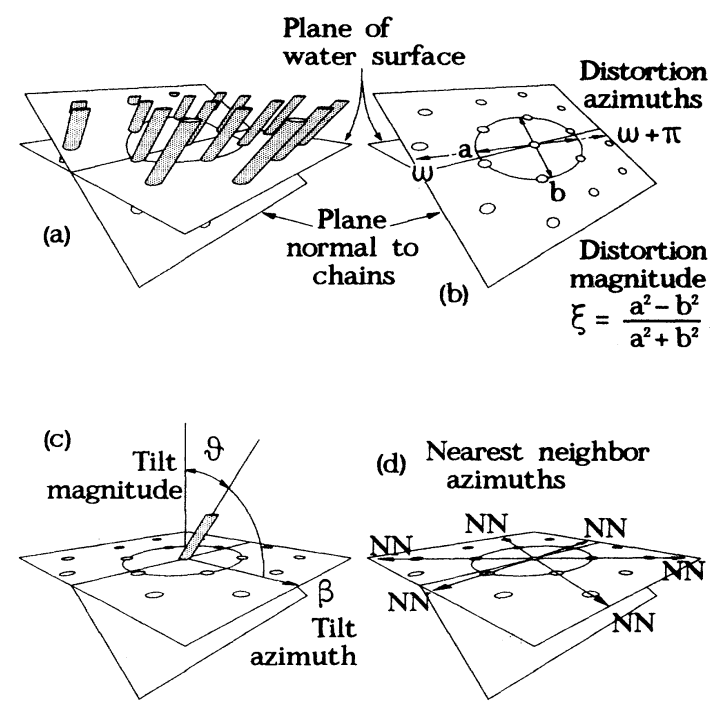

FIG. 5. (a) A perspective cartoon of a two-dimensional lattice of rodlike molecules including the plane of the molecular heads and the plane normal to the chains. The parameters of Table II are illustrated by (b) a cutaway view showing the magnitude $\xi$ and azimuth $\omega$ of distortion normal to the chains; (c) a cutaway view showing the magnitude $\theta$ and azimuth $\beta$ of the molecular tilt; and (d) a cutaway view showing the six nearest neighbor azimuths, a weighted average of which is the origin for the measurement of $\omega$ and $\beta$. marked, but significant discontinuities in the other three curves are also apparent, indicative of a phase transition.

Table II shows parameters extracted from the peak coordinates given in Table I. $A_{\mathrm{xs}}$ is the cross-sectional area normal to the chains; $\theta$ and $\beta$ are the magnitude and direction of the molecular tilt with respect to the average NN direction; $\xi$ and $\omega$ are the magnitude and direction of the distortion perpendicular to the chains, defined in terms of the ellipse drawn through each of the six nearest neighbors of a given molecule. If its major and minor axes are $a$ and $b$, respectively, then $\xi=\left(a^{2}-b^{2}\right) /\left(a^{2}+b^{2}\right)$, while $\omega$ is the direction of its major axis, with respect to the same average NN direction [21]. Figure 5 shows these quantities on a perspective view of a cartoon monolayer lattice of rodlike molecules.

\section{DISCUSSION}

In all recent GID studies of amphiphilic monolayers in which diffraction peaks were observed, it has been assumed that the molecules are essentially packed in a two-dimensional lattice with a coordination number of six. This is justified because it is the dominant mode of packing for aliphatic chain derivatives [23]. The present results are consistent with this idea. The vertical peak widths are always approximately equal to $0.25 \mathrm{rad} \AA^{-1}$, implying a correlation length in the $z$ direction of $25 \AA$.' This is essentially equal to the thickness of the monolayer, indicating that the molecules are behaving like rigid rods. In the general case, a lattice with sixfold coordination gives rise to three low-order peaks. Symmetries of the lattice may reduce the number observed in a powder pattern to two or even one, by causing them to become degenerate, i.e., to overlap.

Again following common practice, the intensities were not fitted directly to the parameters of a lattice. Instead, the $x, y$, and $z$ coordinates of the peaks were fitted. The choice of possible coordinates could, however, be restricted because a two-dimensional lattice is fully described by three parameters (two sides of the unit cell and the included angle) and the molecular tilt is fully described by two (the tilt magnitude and direction with respect to the unit cell vector $a$ ). In the general case, three peak positions are described by six parameters, so that there must be a relationship between them, which is readily shown to be $Q_{z 1}=Q_{z 2}+Q_{z 3}$. The simplified relationships in the case of two peaks result from setting $Q_{z 3}=Q_{z 1}$ (NN tilt) 
or $Q_{z 3}=Q_{z 2}$ (NNN tilt).

All the observed peaks have an appreciable in-plane width $\Delta Q_{x y}$. Although an ideal crystal in two dimensions does not have true long-range order, but only quasi-longrange order, leading to power-law-profile diffraction peaks instead of delta functions, the FHWM of its peaks should still be essentially equal to the diffractometer resolution. This has been found to be the case in the CS phase, which in eicosanoic acid monolayers occurs [24] at high surface pressures below $15^{\circ} \mathrm{C}$. Peak broadening is often interpreted as arising from a polycrystalline texture. In this interpretation, the average domain size is extremely small, of the order of tens of lattice spacings. However, it is known from optical observations [25] that domain sizes are much larger, of the order of millimeters, and that the crystal orientation may vary continuously within the domains. This behavior is characteristic for hexatic mesophases. In many respects hexatics resemble crystals, but they differ in that the hexatic lattice possesses a finite density of thermally generated dislocations.

In light of the identification of the present phases as hexatic, in-plane peak widths are characteristic for, and provide information about, the lattice defects of the thermodynamic ground state. It would be interesting to interpret the significant variations of the $A$ peak width in the high-pressure series. Unfortunately, while some progress has been made in interpreting the peak profiles of a hexatic with upright cylindrical molecules [26], the analysis is still incapable of handling the cases of tilt and herringbone order.

Three distinct peaks were observed at the higher surface pressure for all temperatures in the range 23.8 to $31.3^{\circ} \mathrm{C}$. This has implications for the monolayer phase symmetry. A hep lattice of untilted rods has six planes of mirror symmetry perpendicular to the water surface, but the fact that intensity maxima occur for nonzero $Q_{z}$ means that the molecules are tilted, ruling out all but the plane containing the molecular axes. The absence of degeneracy rules out even this one, so that the structure is chiral. The monolayer symmetries at the two pressures are therefore different, and there must be a phase transition at an intermediate value of surface pressure.

This observation of different phase symmetries between the low-pressure and high-pressure conditions is supported by the previous report of an isotherm kink in this region of the generalized phase diagram of long-chain amphiphiles. It was most distinct in mixtures of docosanoic acid and ethyl eicosanoate, but there were traces of its occurrence above $28^{\circ} \mathrm{C}$ in pure docosanoic acid [27,28]. The kink occurs at approximately $10 \mathrm{mN} / \mathrm{m}$, almost independent of temperature over the range $28-35^{\circ} \mathrm{C}$. Considering that similar features in eicosanoic acid occur at temperatures approximately $10^{\circ} \mathrm{C}$ lower, this fits well with the present observations. It indicates that the present high-pressure phase is the $L_{1}^{\prime}$ phase of Bibo, Knobler, and Peterson [27,28].

Two other distinct triclinic subcell packings of monolayers have been reported. The one reported by Viswanathan, Zasadinski, and Schwartz [14] in monolayers on a solid support has a cross section normal to the chain axes of less than $18.4 \AA^{2}$ and a distortion of more than 0.20 in a near-NNN direction. The chiral monolayer structure reported by Leveiller et al. [29] shows a cross section of $18.6 \AA^{2}$ and a distortion of 0.15 in a near-NN direction. Packings with essentially identical subcells of both types are known for crystals of bulk aliphatic chain derivatives [23]. Both subcells are nearly mirror symmetric, and the monolayer chirality is related to the tilt in a nonsymmetry direction. The chain cross section in the present case is greater than $19.8 \AA^{2}$ and the distortion is less than 0.03 , identifying it as a rotator phase. There is, in fact, a close correspondence with the parameters of the chiral $R_{I I I}$ phase of the long-chain alkanes $[30,31]$.

The present results indicate a limit to the validity of Pasteur's argument for racemic segregation. In this argument, the lack of mirror symmetry in a molecular packing indicates that packing consists only of a pure enantiomer. Certainly, Pasteur's argument is quite valid for three-dimensional crystalline solids. Unfortunately, it is clear from the present results, where the constituent molecules are not chiral, that it is inapplicable for twodimensional mesophases. We concur with Viswanathan et al. [14] that the occurrence of a chiral phase cannot be interpreted as proof of racemic segregation. In racemic mesophase systems, the amounts of each enantiomer in each individual phase will not be equal, but this imbalance may be far from complete segregation.

The present results on a metal-free system indicate that metal ions are not essential for chiral symmetry breaking. In fact, a closely related structure is found in the alkanes that do not even have headgroups. Clearly, the metal ions influence the packing adopted by the system, but they are not an essential component.

Figure 4 provides evidence of the occurrence of a second phase transition. We tentatively identify it with the herringbone-rotator transition predicted by Kaganer, Indenbom, and Loginov [32,33]. Their Landau analysis of monolayer phases indicated that there should be two distinct NN-tilted phases, symbolized as $L_{2 h}$ and $L_{2 d}$, which should show herringbone order and disorder, respectively, of the molecular zigzag planes. The transition between them should occur at approximately constant temperature, almost independent of surface pressure. Such a constant-temperature feature is apparent in a number of reported phase diagrams [27,34]. Figure 6 shows a possible topology, consistent with the isotherm data, for this region of the $(\pi, \mathbf{T})$ phase diagram of eicosanoic acid monolayers.

It may appear incorrect to identify the structure at temperatures just above the transition as a rotator phase because the distortion $\xi$ of its unit cell differs from zero. However this is exactly the situation observed by Levelut [35] in bulk smectic- $B H$ phases at temperatures just above the transition to the smectic- $E$ phase. By analysis of the diffraction pattern, Levelut was able to demonstrate the existence of an extremely fine and rapidly fluctuating pattern of herringbone-ordered domains with a characteristic size of up to 40 lattice spacings. The correlations of these herringbone fluctuations decay exponentially with increasing separation, meaning that the order is only short range, so that the macroscopic phase sym- 


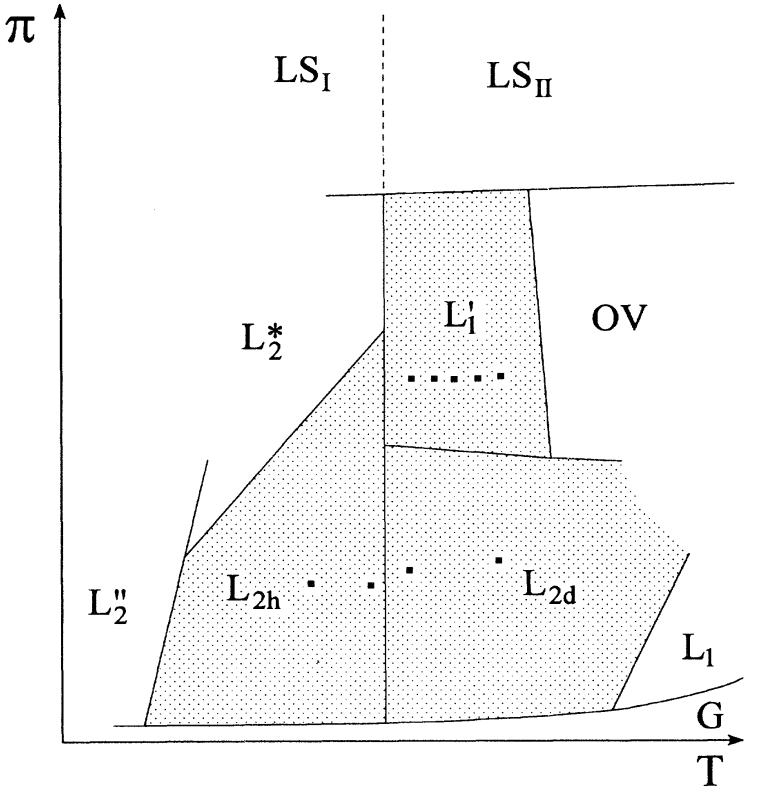

FIG. 6. Possible topological relationships in the $(\pi, T)$ phase diagram between the three phases observed in the present investigation, shown shaded, and known adjoining phases. The dots indicate the approximate relative positions of the measurement points.

metry is hexagonal. However, the fluctuations give rise to structure in the powder pattern similar to that expected from a polycrystalline texture of a rectangular crystalline packing. Unlike a polycrystalline texture, the nanodomains of herringbone order in the smectic- $B H$ phase are in thermal equilibrium. The finite linewidth is a property of the thermodynamic ground state, and there is no tendency to anneal toward a state of long-range translational order. The present observation of nonzero $\xi$ in a tilted rotator phase parallels the observations of Shih et al. [36] in upright monolayer phases.

The reproducibility of the present measurements is as good as the best normal practice. The peak intensities were affected by slow beam damage: the diffracted intensities just after moving the trough to expose a new area of monolayer were typically $10 \%$ more intense than just before. It has been assumed that the present measurements yield the intensities expected from a powder pattern with perfect orientational disorder. Because the trough could not be rotated, it was impossible to estimate the extent of departure from this assumption.

It is conceivable that the peak positions might also vary. This has in fact been claimed by Schlossman et al. [37], who observed a tetracosanoic acid monolayer at constant area; under one condition they reported progressive variation of the structure from tilted to upright to tilted again with characteristic times of days. This shift of peak positions must be distinguished from the universally observed relaxation of area at constant surface pressure, which is most probably related to a morphology change ("sintering"). The present monolayer was held at each surface condition of temperature and surface pressure for at most two hours.

There are nevertheless two arguments for the significance of the present results. First, even in experiments on dedicated equipment, it is unusual to observe the one monolayer for more than a few hours. The phase diagram of tetracosanoic acid reported by Stenhagen [24], showing two triple points close to room temperature, is consistent with the initial behavior observed by Schlossman et al. [37]. Hence the short-term behavior is reproducible and is of considerable relevance to normal monolayer work. Second, there are grounds for believing that the long-term results of Schlossman et al. are not characteristic for a monolayer of pure tetracosanoic acid. Their subphase was not purified immediately before use but was transported to the synchrotron in borosilicate glass containers [38], in spite of a report by Albrecht [39] that the properties of monolayers change over a period of hours on such a subphase but not on pure water. Peterson and Russell [40] found related slow changes in a monolayer on a subphase in contact with soda-lime glass, which in a subsequent publication [41] were traced to the action of metal ions.

While the solubility of borosilicate glass is of the order of $1 \mu \mathrm{M}$, it should be noted that Langmuir and Schaeffer [42] reported distinct differences in the properties of fatty acid monolayers after minutes in the presence of $50 \mathrm{nM}$ $\mathrm{Cu}^{2+}$ or $20 \mathrm{nM} \mathrm{Al}^{3+}$ solutions, at which concentrations the time for diffusion of a monolayer to the surface is measured in hours. This can be explained by strong complexation [43] between the metal and the headgroups. The specific structures of some of these complexes have been determined $[44,45]$. To conclude, it is possible that the relaxation times reported by Schlossman and coworkers $[37,38]$ merely represent the diffusion to the surface of some constituent of borosilicate glass, probably metal ions. In fact, in all studies in which the monolayer is kept at constant surface pressure and the deionized subphase has been in contact only with fluoropolymers, there has been no relaxation of diffraction peak positions.

\section{CONCLUSION}

It is ironic that, since the fatty acids are the "classical" amphiphiles, investigated by a variety of methods for over a century, they are often believed to be so simple as to be of little interest. The present measurements, covering a relatively limited range of surface pressure and temperature, have revealed three distinct phases in a region previously believed to be uniform. It is only four years since the GID confirmation of the many phase distinctions reported by Stenhagen in docosanoic acid in the vicinity of room temperature, and only two years since the discovery of the Overbeck-Möbius phase at room temperature in the most extensively studied fatty acid of all, octadecanoic. We believe that the fatty acids and other simple amphiphiles will reveal yet further subtlety of behavior. There is evidence that this, or similar, behavior is generic for all aliphatic chain compounds, so that the present discoveries have wide implications, most likely including the sensitivity of biological membranes to their environment. 


\section{ACKNOWLEDGMENTS}

This work received financial support from the Fonds Henri de Rothschild, the French Ministère des Affaires Étrangères, and the Danish Natural Science Research
Council (Grant 3.70.01-08/93). The authors would like to thank Dr. V. M. Kaganer, Professor C. M. Knobler, Professor P. S. Pershan, and Professor H. Möhwald for useful discussions, and HASYLAB at DESY, Hamburg, Germany, for synchrotron beam time.
[1] Structures and Properties of Cell Membranes, edited by G. Benga (CRC, Boca Raton, FL, 1985), Vol. 3.

[2] Langmuir-Blodgett Films, edited by G. G. Roberts (Plenum, New York, 1990).

[3] D. R. Nelson and B. I. Halperin, Phys. Rev. B 19, 2457 (1979).

[4] D. R. Nelson and B. I. Halperin, Phys. Rev. B 21, 5212 (1980).

[5] S. Ställberg-Stenhagen and E. Stenhagen, Nature (London) 156, 239 (1945).

[6] B. Lin, M. C. Shih, T. M. Bohanon, G. E. Ice, and P. Dutta, Phys. Rev. Lett. 65, 191 (1990).

[7] R. M. Kenn, C. Böhm, A. M. Bibo, I. R. Peterson, K. Kjaer, and J. Als-Nielsen, J. Phys. Chem. 95, 2092 (1991).

[8] T. M. Fischer, R. Bruinsma, and C. M. Knobler, Phys. Rev. E 50, 413 (1994).

[9] J. Ruiz-Garcia, X. Qiu, M. W. Tsao, G. Marshall, C. M. Knobler, G. A. Overbeck, and D. Möbius, J. Phys. Chem. 97, 6955 (1993).

[10] M. Lundquist, Ark. Kemi 17, 183 (1961).

[11] M. Lundquist, Ark. Kemi 23, 299 (1965).

[12] E. M. Arnett and J. M. Gold, J. Am. Chem. Soc. 104, 636 (1982).

[13] C. J. Eckhardt, N. M. Peachey, D. R. Swanson, J. M. Takacs, M. A. Khan, X. Gong, J.-H. Kim, J. Wang, and R. A. Uphaus, Nature (London) 362, 614 (1993).

[14] R. Viswanathan, J. A. Zasadinski, and D. K. Schwartz, Nature (London) 368, 440 (1994).

[15] K. Kjaer, in Annual Progress Report of the Department of Solid State Physics, edited by J. Skov Pedersen, B. Lebech, and P.-A. Lindgård, Ris $\varnothing$ National Laboratory, Roskilde, Denmark Ris $\varnothing-R-660(E N), 1992$, pp. 130 and 131.

[16] K. Kjaer, HASYLAB Annual Report, 1992, pp. 589 and 590 (unpublished).

[17] K. Kjaer, Experimental Stations at HASYLAB, 1994, pp. 88 and 89 (unpublished).

[18] F. Leveiller, D. Jacquemain, L. Leiserowitz, K. Kjaer, and J. Als-Nielsen, J. Phys. Chem. 96, 10380 (1992).

[19] S. K. Sinha, E. B. Sirota, S. Garoff, and H. B. Stanley, Phys. Rev. B 38, 2297 (1988).

[20] M. Durbin, A. Malik, R. Ghaskadvi, M. C. Shih, P. Zschack, and P. Dutta, J. Phys. Chem. 98, 1753 (1994).

[21] V. M. Kaganer, I. R. Peterson, R. M. Kenn, M. C. Shih, M. Durbin, and P. Dutta, J. Chem. Phys. 102, 9412 (1995).

[22] R. M. Kenn, C. Böhm, H. Möhwald, K. Kjaer, and J. Als-Nielsen, in Surface $X$-Ray and Neutron Scattering, edited by H. Zabel and I. K. Robinson (Springer, Berlin, 1992).

[23] E. Segerman, Acta Cryst. 19, 789 (1965).

[24] E. Stenhagen, in Determination of Organic Structures by Physical Methods, edited by E. A. Braude and F. C. Nachod (Academic, New York, 1955).

[25] G. A. Overbeck and D. Möbius, J. Phys. Chem. 97, 7999 (1993).

[26] I. R. Peterson and V. M. Kaganer, Phys. Rev. Lett. 73, 102 (1994).

[27] A. M. Bibo, C. M. Knobler, and I. R. Peterson, J. Phys. Chem. 95, 5591 (1991).

[28] A. M. Bibo and I. R. Peterson, Thin Solid Films 210/211, 515 (1992).

[29] F. Leveiller, D. Jacquemain, M. Lahav, L. Leiserowitz, M. Deutsch, K. Kjaer, and J. Als-Nielsen, Science 252, 1532 (1991).

[30] E. B. Sirota, H. E. King, Jr., D. M. Singer, and H. H. Shao, J. Chem. Phys. 98, 5809 (1993).

[31] I. R. Peterson and R. M. Kenn, Langmuir 10, 4645 (1994).

[32] V. M. Kaganer and V. L. Indenbom, J. Phys. (France) II 3, 813 (1993).

[33] V. M. Kaganer and E. B. Loginov, Phys. Rev. Lett. 71, 2599 (1993).

[34] M. C. Shih, M. K. Durbin, A. Malik, P. Zshack, and P. Dutta, J. Chem. Phys. 101, 9132 (1994).

[35] A. M. Levelut, J. Phys. (Paris) Colloq. 37, C3-51 (1976).

[36] M. C. Shih, T. M. Bohanon, J. M. Mikrut, P. Zschack, and P. Dutta, Phys. Rev. A 45, 5734 (1992).

[37] M. L. Schlossman, D. K. Schwartz, P. S. Pershan, E. H. Kawamoto, G. J. Kellogg, and S. Lee, Phys. Rev. Lett. 66, 1599 (1991).

[38] D. K. Schwartz, M. L. Schlossman, and P. S. Pershan, J. Chem. Phys. 96, 2356 (1992).

[39] O. Albrecht, Thin Solid Films 99, 227 (1983).

[40] I. R. Peterson and G. J. Russell, Philos. Mag. 49A, 463 (1984).

[41] G. Veale and I. R. Peterson, J. Colloid. Interface Sci. 103, 178 (1985).

[42] I. Langmuir and V. J. Schaeffer, J. Am. Chem. Soc. 59, 2400 (1937).

[43] H. T. S. Britton and F. H. Meek, J. Chem. Soc. 2831 (1931).

[44] A. B. Blake and L. R. Fraser, J. Chem. Soc. Dalton Trans. 193 (1975).

[45] L. E. Orgel, Nature (London) 187, 504 (1960). 